\title{
Exploring Text and Image Features to Classify Images in Bioscience Lit- erature
}

\author{
Barry Rafkind \\ DVMM Group \\ Columbia University \\ New York, NY 10027 \\ Barryr \\ dee.columbia.edu
}

\author{
Minsuk Lee \\ Department of Health Sci- \\ ences \\ University of Wisconsin- \\ Milwaukee \\ Milwaukee, WI 53201 \\ Minsuk. Lee \\ @gmail.com
}

\author{
Shih-Fu Chang \\ DVMM Group \\ Columbia University \\ New York, NY 10027 \\ Sfchang \\ dee.columbia.edu
}

\author{
Hong Yu \\ Department of Health Sci- \\ ences \\ University of Wisconsin- \\ Milwaukee \\ Milwaukee, WI 53201 \\ Hong. Yu @uwm.edu
}

\begin{abstract}
A picture is worth a thousand words. Biomedical researchers tend to incorporate a significant number of images (i.e., figures or tables) in their publications to report experimental results, to present research models, and to display examples of biomedical objects. Unfortunately, this wealth of information remains virtually inaccessible without automatic systems to organize these images. We explored supervised machine-learning systems using Support Vector Machines to automatically classify images into six representative categories based on text, image, and the fusion of both. Our experiments show a significant improvement in the average Fscore of the fusion classifier $(73.66 \%)$ as compared with classifiers just based on image $(50.74 \%)$ or text features $(68.54 \%)$.
\end{abstract}

\section{Introduction}

A picture is worth a thousand words. Biomedical researchers tend to incorporate a significant number of figures and tables in their publications to report experimental results, to present research models, and to display examples of biomedical objects (e.g., cell, tissue, organ and other images). For example, we have found an average of $5.2 \mathrm{im}-$ ages per biological article in the journal Proceedings of the National Academy of Sciences (PNAS). We discovered that $43 \%$ of the articles in the medical journal The Lancet contain biomedical images. Physicians may want to access biomedical images reported in literature for the purpose of clinical education or to assist clinical diagnoses. For example, a physician may want to obtain images that illustrate the disease stage of infants with Retinopathy of Prematurity for the purpose of clinical diagnosis, or to request a picture of erythema chronicum migrans, a spreading annular rash that appears at the site of tick-bite in Lyme disease. Biologists may want to identify the experimental results or images that support specific biological phenomenon. For example, Figure 1 shows that a transplanted progeny of a single multipotent stem cell can generate sebaceous glands.

Organizing bioscience images is not a new task. Related work includes the building of domainspecific image databases. For example, the Protein Data Bank (PDB) ${ }^{1}$ (Sussman et al., 1998) stores 3-D images of macromolecular structure data. WebPath $^{2}$ is a medical web-based resource that has been created by physicians to include over 4,700 gross and microscopic medical images. Textbased image search systems like Google ignore image content. The SLIF (Subcellular Location Image Finder) system (Murphy et al., 2001; Kou et al., 2003) searches protein images reported in literature. Other work has explored joint text-image features in classifying protein subcellular location images (Murphy et al., 2004). The existing systems, however, have not explored approaches that automatically classify general bioscience images into generic categories.

\footnotetext{
${ }^{1}$ http://www.rcsb.org/pdb/

${ }^{2}$ http://www-medlib.med.utah.edu/WebPath/webpath.html
} 
Classifying images into generic categories is an important task that can benefit many other natural language processing and image processing tasks. For example, image retrieval and question answering systems may return "Image-of-Thing" images (e.g., Figure 1), not the other types (e.g., Figure $2 \sim 5)$, to illustrate erythema chronicum migrans. Biologists may examine "Gel" images (e.g., Figure 2), rather than "Model" (e.g., Figure 4) to access specific biological evidence for molecular interactions. Furthermore, a generic category may ease the task of identifying specific images that may be sub-categories of the generic category. For example, a biologist may want to obtain an image of a protein structure prediction, which might be a subcategory of "Model" (Figure 4), rather than an image of x-ray crystallography that can be readily obtained from the PDB database.

This paper represents the first study that defines a generic bioscience image taxonomy, and explores automatic image classification based on the fusion of text and image classifiers.

Gel-Image consists of gel images such as Northern (for DNA), Southern (for RNA), and Western (for protein). Figure 2 shows an example.

Graph consists of bar charts, column charts, line charts, plots and other graphs that are drawn either by authors or by a computer (e.g., results of patch clamping). Figure 3 shows an example.

Image-of-Thing refers to images of cells, cell components, tissues, organs, or species. Figure 1 shows an example.

Mix refers to an image (e.g., Figure 5) that incorporates two or more other categories of images.

Model: A model may demonstrate a biological process, molecular docking, or an experimental design. We include as Model any structure (e.g., chemical, molecular, or cellular) that is illustrated by a drawing. We also include gene or protein sequences and sequence alignments, as well as phylogenetic trees in this category. Figure 4 shows one example.

Table refers to a set of data arranged in rows and columns.

Table 1. Bioscience Image Taxonomy

\section{Image Taxonomy}

We downloaded from PubMed Central a total of 17,000 PNAS full-text articles (years 1995-2004), which contain a total of 88,225 images. We manually examined the images and defined an image taxonomy (as shown in Table 1) based on feedback from physicians. The categories were chosen to maintain balance between coherence of content in each category and the complexity of the taxonomy. For example, we keep images of biological objects (e.g., cells, tissues, organs etc) in one single category in this experiment to avoid over decomposition of categories and insufficient data in individual categories. Therefore we stress principled approaches for feature extraction and classifier design. The same fusion classification framework can be applied to cases where each category is further refined to include subclasses.
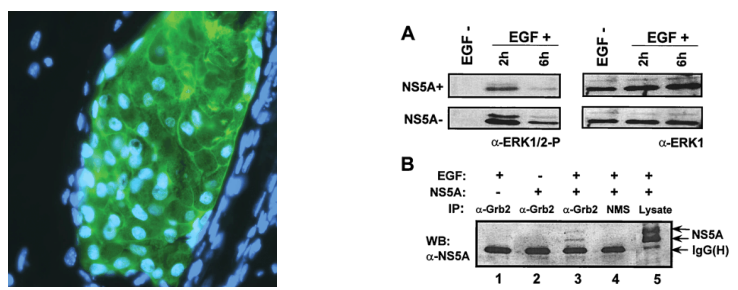

Figure 1. Image of_Thing ${ }^{3}$ Figure 2. Gel image $^{4}$
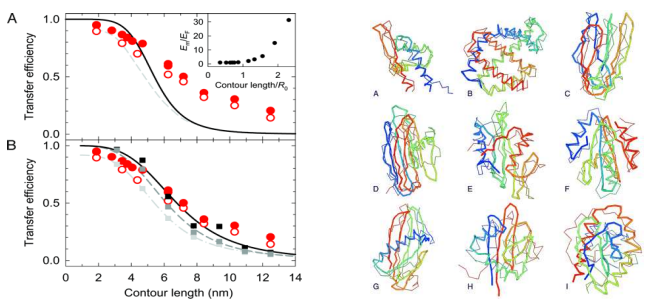

Figure 3. Graph image ${ }^{5}$ Figure 4. Model image ${ }^{6}$

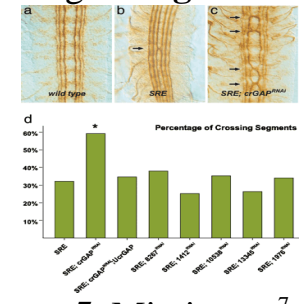

Figure 5. Mix image ${ }^{7}$

\footnotetext{
${ }^{3}$ This image appears in the cover page of PNAS 102 (41): 14477 - 14936.

${ }^{4}$ The image appears in the article (pmid=10318918)

${ }^{5}$ The image appears in the article (pmid $=15699337$ )

${ }^{6}$ The image appears in the article $(\mathrm{pmid}=11504922)$

${ }^{7}$ The image appears in the article $(\mathrm{pmid}=15755809)$
} 


\section{Image Classification}

We explored supervised machine-learning methods to automatically classify images according to our image taxonomy (Table 1). Since it is straightforward to distinguish table separately by applying surface cues (e.g., "Table" and "Figure"), we have decided to exclude it from our experiments.

\subsection{Support Vector Machines}

We explored supervised machine-learning systems using Support Vector Machines (SVMs) which have shown to out-perform many other supervised machine-learning systems for text categorization tasks (Joachims, 1998). We applied the freely available machine learning MATLAB package The Spider to train our SVM systems (Sable and Weston, 2005; MATLAB). The Spider implements many learning algorithms including a multi-class SVM classifier which was used to learn our discriminative classifiers as described below in section 3.4.

A fundamental concept in SVM theory is the projection of the original data into a highdimensional space in which separating hyperplanes can be found. Rather than actually doing this projection, kernel functions are selected that efficiently compute the inner products between data in the high-dimensional space. Slack variables are introduced to handle non-separable cases and this requires an upper bound variable, $\mathrm{C}$.

Our experiments considered three popular kernel function families over five different variants and five different values of $\mathrm{C}$. The kernel function implementations are explained in the software documentation. We considered kernel functions in the forms of polynomial, radial basis function, and Gaussian. The adjustable parameter for polynomial functions is the order of the polynomial. For radial basis function and Gaussian functions, sigma is the adjustable parameter. A grid search was performed over the adjustable parameter for values 1 to 5 and for values of $\mathrm{C}$ equal to $\left[10^{\wedge} 0,10^{\wedge} 1,10^{\wedge} 2,10^{\wedge} 3\right.$, $\left.10^{\wedge} 4\right]$.

\subsection{Text Features}

Previous work in the context of newswire image classification show that text features in image captions are efficient for image categorization (Sable, 2000, 2002, 2003). We hypothesize that image captions provide certain lexical cues that efficiently represent image content. For example, the words "diameter", "gene-expression", "histogram", "lane", "model", "stained", "western", etc are strong indicators for image classes and therefore can be used to classify an image into categories. The features we explored are bag-of-words and ngrams from the image captions after processing the caption text by the Word Vector Tool (Wurst).

\subsection{Image Features}

We also investigated image features for the tasks of image classification. We started with four types of image features that include intensity histogram features, edge-direction histogram features, edgebased axis features, and the number of 8-connected regions in the binary-valued image obtained from thresholding the intensity.

The intensity histogram was created by quantizing the gray-scale intensity values into the range 0 255 and then making a 256-bin histogram for these values. The histogram was then normalized by dividing all values by the total sum. For the purpose of entropy calculations, all zero values in the histogram are set to one. From this adjusted, normalized histogram, we calculated the total entropy as the sum of the products of the entries with their logarithms. Additionally, the mean, 2nd moment, and 3 rd moment are derived. The combination of the total entropy, mean, 2nd, and 3rd moments constitute a robust and concise representation of the image intensity.

Edge-Direction Histogram (Jain and Vailaya, 1996) features may help distinguish images with predominantly straight lines such as those found in graphs, diagrams, or charts from other images with more variation in edge orientation. The EDH begins by convolving the gray-scale image with both $3 \times 3$ Sobel edge operators (Jain, 1989). One operator finds vertical gradients while the other finds horizontal gradients. The inverse tangent of the ratio of the vertical to horizontal gradient yields continuous orientation values in the range of - pi to + pi. These values are subsequently converted into degrees in the range of 0 to 179 degrees (we consider 180 and 0 degrees to be equal). A histogram is counted over these 180 degrees. Zero values in the histogram are set to one in order to anticipate entropy calculations and then the modified histogram is normalized to sum to one. Finally, the total 
entropy, mean, 2nd and 3rd moments are extracted to summarize the EDH.

The edge-based axis features are meant to help identify images containing graphs or charts. First, Sobel edges are extracted above a sensitivity threshold of 0.10 from the gray-scale image. This yields a binary-valued intensity image with 1's occurring in locations of all edges that exceed the threshold and 0's occurring otherwise. Next, the vertical and horizontal sums of this intensity image are taken yielding two vectors, one for each axis. Zero values are set to one to anticipate the entropy calculations. Each vector is then normalized by dividing each element by its total sum. Finally, we find the total entropy, mean, 2nd , and 3rd moments to represent each axis for a total of eight axis features.

The last image feature under consideration was the number of 8-connected regions in the binaryvalued, thresholded Sobel edge image as described above for the axis features. An 8-connected region is a group of edge pixels for which each member touches another member vertically, horizontally, or diagonally in the eight adjacent pixel positions surrounding it. The justification for this feature is that the number of solid regions in an image may help separate classes.

A preliminary comparison of various combinations of these image features showed that the intensity histogram features used alone yielded the best classification accuracy of approximately $54 \%$ with a quadratic kernel SVM using an upper slack limit of $\mathrm{C}=10^{\wedge} 4$.

\subsection{Fusion}

We integrated both image and text features for the purpose of image classification. Multi-class SVM's were trained separately on the image features and the text features. A multi-class SVM attempts to learn the boundaries of maximal margin in feature space that distinguishes each class from the rest. Once the optimal image and text classifiers were found, they were used to process a separate set of images in the fusion set. We extracted the margins from each data point to the boundary in feature space.

Thus, for a five-class classifier, each data point would have five associated margins. To make a fair comparison between the image-based classifier and the text-based classifier, the margins for each data point were normalized to have unit magnitude. So, the set of five margins for the image classifier constitutes a vector that then gets normalized by dividing each element by its L2 norm. The same is done for the vector of margins taken from the text classifier. Finally, both normalized vectors are concatenated to form a 10-dimensional fusion vector. To fuse the margin results from both classifiers, these normalized margins were used to train another multi-class SVM.

A grid search through parameter space with cross validation identified near-optimal parameter settings for the SVM classifiers. See Figure 6 for our system flowchart.

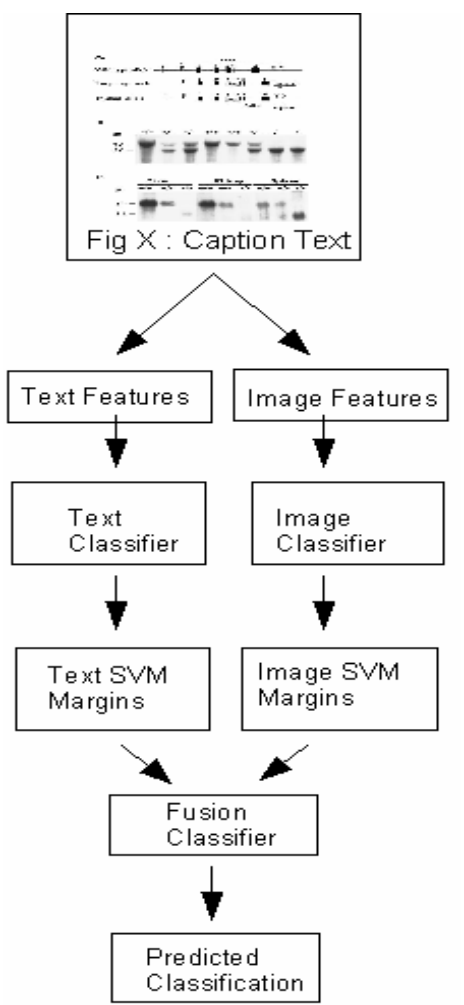

Figure 6. System Flow-chart

\subsection{Training, Fusion, and Testing Data}

We randomly selected a subset of 554 figure images from the total downloaded image pool. One author of this paper is a biologist who annotated figures under five classes; namely, Gel_Image (102), Graph (179), Image_of_Thing (64), Mix (106), and Model (103).

These images were split up such that for each category, roughly a half was used for training, a quarter for fusion, and a quarter for testing (see Figure 7). The training set was used to train classi- 
fiers for the image-based and text-based features. The fusion set was used to train a classifier on top of the results of the image-based and text-based classifiers. The testing set was used to evaluate the final classification system.

For each division of data, 10 folds were generated. Thus within the training and fusion data sets, there are 10 folds which each have a randomized partitioning into $90 \%$ for training and $10 \%$ for testing. The testing data set did not need to be partitioned into folds since all of it was used to test the final classification system. (See Figure 8).

In the 10 -fold cross-validation process, a classifier is trained on the training partition and then measured for accuracy (or error rate) on the testing partition. Of the 10 resulting algorithms, the one which performs the best is chosen (or just one which ties for the best accuracy).

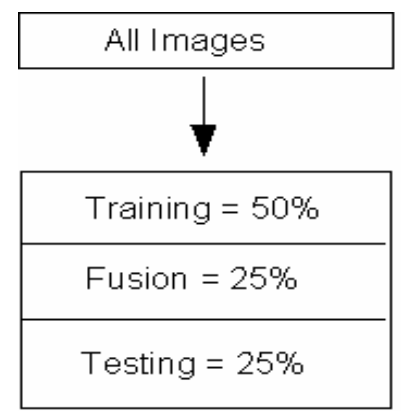

Figure 7. Image-set Divisions

\subsection{Evaluation Metrics}

We report the widely used recall, precision, and Fscore (also known as F-measure) as the evaluation metrics for image classification. Recall is the total number of true positive predictions divided by the total number of true positives in the set (true pos + false neg). Precision is the fraction of the number of true positive predictions divided by the total number of positive predictions (true pos + false pos). F-score is the harmonic mean of recall and precision equal to (C. J. van Rijsbergen, 1979): $2 *$ precision $*$ recall $/($ precision + recall $)$

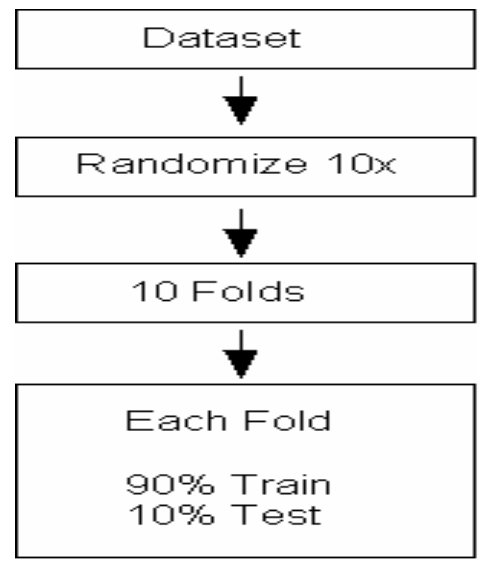

Figure 8. Partitioning Method for Training and Fusion Datasets

\section{Experimental Results}

Table 2 shows the Confusion Matrix for the image feature classifier obtained from the testing part of the training data. The actual categories are listed vertically and predicted categories are listed horizontally. For instance, of 26 actual GEL images, 18 were correctly classified as GEL, 4 were misclassified as GRAPH, 2 as IMAGE_OF_THING, 0 as MIX, and 2 as MODEL.

\begin{tabular}{|l|l|l|l|l|l|}
\hline Actual & \multicolumn{5}{l}{ Predicted Categories } \\
\hline & Gel & Graph & Thing & Mix & Model \\
\hline Gel & 18 & 4 & 2 & 0 & 2 \\
\hline Graph & 3 & 39 & 0 & 1 & 1 \\
\hline Img_Thing & 1 & 1 & 12 & 2 & 0 \\
\hline Mix & 4 & 17 & 0 & 3 & 3 \\
\hline Model & 8 & 13 & 0 & 1 & 3 \\
\hline
\end{tabular}

Table 2. Confusion Matrix for Image Feature Classifier

A near-optimal parameter setting for the classifier based on image features alone used a polynomial kernel of order 2 and an upper slack limit of $C$ $=10^{\wedge} 4$. Table 3 shows the performance of image classification with image features. True Positives, False Positives, False Negatives, Precision = $\mathrm{TP} /(\mathrm{TP}+\mathrm{FP})$, Recall $=\mathrm{TP} /(\mathrm{TP}+\mathrm{FN})$, and F-score $=$ $2 *$ Precision $*$ Recall / (Precision + Recall). According to the F-score scores, this classifier does best on distinguishing IMAGE_OF_THING images. The overall accuracy $=$ sum of true positives $/$ total number of images $=(18+39+12+3+3) / 138=$ $75 / 138=54 \%$. This can be compared with the baseline of $(3+39+1+1) / 138=32 \%$ if all images 
were classified as the most popular category, GRAPH. Clearly, the image-based classifier does best at recognizing IMAGE_OF_THING figures.

\begin{tabular}{|l|l|l|l|l|l|l|}
\hline Category & TP & FP & FN & Prec. & Recall & Fscore \\
\hline Gel & 18 & 16 & 8 & 0.529 & 0.692 & 0.600 \\
\hline Graph & 39 & 35 & 5 & 0.527 & 0.886 & 0.661 \\
\hline Img_Thing & 12 & 2 & 4 & 0.857 & 0.750 & 0.800 \\
\hline Mix & 3 & 4 & 10 & 0.429 & 0.231 & 0.300 \\
\hline Model & 3 & 6 & 22 & 0.333 & 0.120 & 0.176 \\
\hline
\end{tabular}

Table 3. Precision, Recall, F-score for Image Classifier

\begin{tabular}{|l|l|l|l|l|l|}
\hline Actual & \multicolumn{6}{|l|}{ Predicted Categories } \\
\hline & Gel & Graph & Thing & Mix & Model \\
\hline Gel & 22 & 2 & 0 & 2 & 0 \\
\hline Graph & 4 & 36 & 0 & 4 & 0 \\
\hline Img_Thing & 0 & 3 & 11 & 1 & 1 \\
\hline Mix & 3 & 9 & 1 & 12 & 2 \\
\hline Model & 3 & 5 & 0 & 3 & 14 \\
\hline
\end{tabular}

Table 4. Confusion Matrix for Caption Text Classifier

\begin{tabular}{|l|l|l|l|l|l|l|}
\hline Category & TP & FP & FN & Prec & Recall & Fscore \\
\hline Gel & 22 & 10 & 4 & 0.688 & 0.845 & 0.758 \\
\hline Graph & 36 & 19 & 8 & 0.655 & 0.818 & 0.727 \\
\hline Img_Thing & 11 & 1 & 5 & 0.917 & 0.688 & 0.786 \\
\hline Mix & 12 & 10 & 15 & 0.545 & 0.444 & 0.489 \\
\hline Model & 14 & 3 & 11 & 0.824 & 0.560 & 0.667 \\
\hline
\end{tabular}

Table 5. Precision, Recall, F-score for Caption

Text Classifier

The text-based classifier excels in finding GEL, GRAPH, and IMAGE_OF_THING images. It achieves an accuracy of $(22+36+11+12+14) / 138=$ $95 / 138=69 \%$.

A near-optimal parameter setting for the fusion classifier based on both image features and text features used a linear kernel with $\mathrm{C}=10$. The corresponding Confusion matrix follows in Table 6.

\begin{tabular}{|l|l|l|l|l|l|}
\hline Actual & \multicolumn{5}{|l|}{ Predicted Categories } \\
\hline & Gel & Graph & Thing & Mix & Model \\
\hline Gel & 23 & 0 & 0 & 3 & 0 \\
\hline Graph & 2 & 37 & 1 & 2 & 2 \\
\hline Img_Thing & 0 & 1 & 15 & 0 & 0 \\
\hline Mix & 2 & 7 & 1 & 14 & 3 \\
\hline Model & 3 & 5 & 0 & 4 & 13 \\
\hline
\end{tabular}

Table 6. Confusion Matrix for Fusion Classifier

\begin{tabular}{|l|l|l|l|l|l|l|}
\hline Category & TP & FP & FN & Prec. & Recall & Fscore \\
\hline Gel & 23 & 7 & 3 & 0.767 & 0.885 & 0.822 \\
\hline Graph & 37 & 13 & 7 & 0.740 & 0.841 & 0.787 \\
\hline Img_Thing & 15 & 2 & 1 & 0.882 & 0.938 & 0.909 \\
\hline Mix & 14 & 9 & 13 & 0.609 & 0.519 & 0.560 \\
\hline Model & 13 & 5 & 12 & 0.722 & 0.520 & 0.605 \\
\hline
\end{tabular}

Table 7. Precision, Recall, F-score for Fusion Classifier

From Table 7, it is apparent that the fusion classifier does best on IMAGE_OF_THING and also performs well on GEL and GRAPH. These are substantial improvements over the classifiers that were based on image or text feature alone. Average F-scores and accuracies are summarized below in Table 8.

The overall accuracy for the fusion classifier $=$ sum of true positives / total number of image $=$ $(23+37+15+14+13) / 138=102 / 138=74 \%$. This can be compared with the baseline of $44 / 138=$ $32 \%$ if all images were classified as the most popular category, GRAPH.

\begin{tabular}{|l|l|l|}
\hline Classifier & Average F-score & Accuracy \\
\hline Image & $50.74 \%$ & $54 \%$ \\
\hline $\begin{array}{l}\text { Caption } \\
\text { Text }\end{array}$ & $68.54 \%$ & $69 \%$ \\
\hline Fusion & $73.66 \%$ & $74 \%$ \\
\hline
\end{tabular}

Table 8. Comparison of Average F-scores and Accuracy among all three Classifiers

\section{Discussion}

It is not surprising that the most difficult category to classify is Mix. This was due to the fact that Mix images incorporate multiple categories of other image types. Frequently, one other image type that appears in a Mix image dominates the image features and leads to its misclassification as the other image type. For example, Figure 9 shows that a Mix image was misclassified as Gel_Image.

This mistake is forgivable because the image does contain sub-images of gel-images, even though the entire figure is actually a mix of gelimages and diagrams. This type of result highlights the overlap between classifications and the difficulty in defining exclusive categories.

For both misclassifications, it is not easy to state exactly why they were classified wrongly based on their image or text features. This lack of 
intuitive understanding of discriminative behavior of SVM classifiers is a valid criticism of the technique. Although generative machine learning methods (such as Bayesian techniques or Graphical Models) offer more intuitive models for explaining success or failure, discriminative models like SVM are adopted here due to their higher performance and ease of use.

Figure 10 shows an example of a MIX figure that was mislabeled by the image classifier as GRAPH and as GEL_IMAGE by the text classifier. However, it was correctly labeled by the fusion classifier. This example illustrates the value of the fusion classifier for being able to improve upon its component classifiers.

\section{Conclusions}

From the comparisons in Table 8, we see that fusing the results of classifiers based on text and image features yields approximately 5\% improvement over the text -based classifier alone with respect to both average F-score and Accuracy. In fact, the F-score improved for all categories except for MODEL which experienced a 6\% drop. The natural conclusion is that the fusion classifier combines the classification performance from the text and image classifiers in a complementary fashion that unites the strengths of both.

\section{Future Work}

To enhance the performance of the text features, one may restrict the vocabulary to functionally important biological words. For example, "phosphorylation" and "3-D" are important words that might sufficiently separate "protein function" from "protein structure".

Further experimentation on a larger image set would give us even greater confidence in our results. It would also expand the diversity within each category, which would hopefully lead to better generalization performance of our classifiers.

Other possible extensions of this work include investigating different machine learning approaches besides SVMs and other fusion methods. Additionally, different sets of image and text features can be explored as well as other taxonomies.

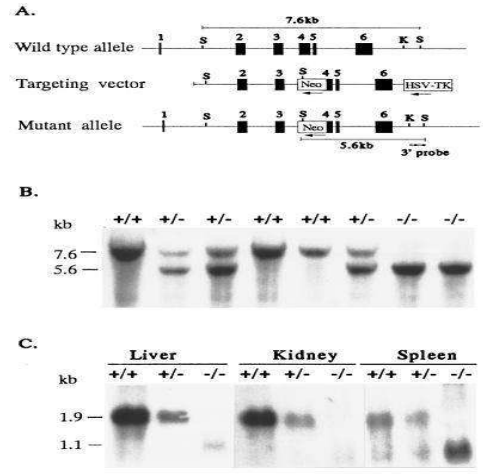

Caption: "The 2.6-kb HincII XhoI fragment containing approximately half of exon 4 and exon 5 and 6 was subcloned between the Neo gene and thymidine kinase (Fig. 1 A). The location of the genomic probe used to screen for homologous recombination is shown in Fig. 1 A. Gene Targeting in Embryonic Stem (ES) Cells and Generation of Mutant Mice. Genomic DNA of resistant clones was digested with SacI and hybridized with the 3 0.9-kb KpnI SacI external probe (Fig. 1 A). Chimeric male offspring were bred to C57BL/6J females and the agouti F1 offspring were tested for transmission of the disrupted allele by Southern blot analysis of SacI-digested genomic DNA by using the 3 external probe (Fig. $1 \mathrm{~A}$ and B). A 360bp region, including the first $134 \mathrm{bp}$ of the 275-bp exon 4, was deleted and replaced with the PGKneo cassette in the reverse orientation (Fig. 1 A). After selection with G418 and gangciclovir, doubly resistant clones were screened for homologous recombination by Southern blotting and hybridization with a 3 external probe (Fig. 1 A). Offspring were genotyped by Southern blotting of genomic tail DNA and hybridized with a 3 external probe (Fig. 1 B). To confirm that HFE / mice do not express the HFE gene product, we performed Northern blot analyses “

Figure 9. Above, caption text and image of a MIX figure mis-classified as GEL_IMAGE by the Fusion Classifier 


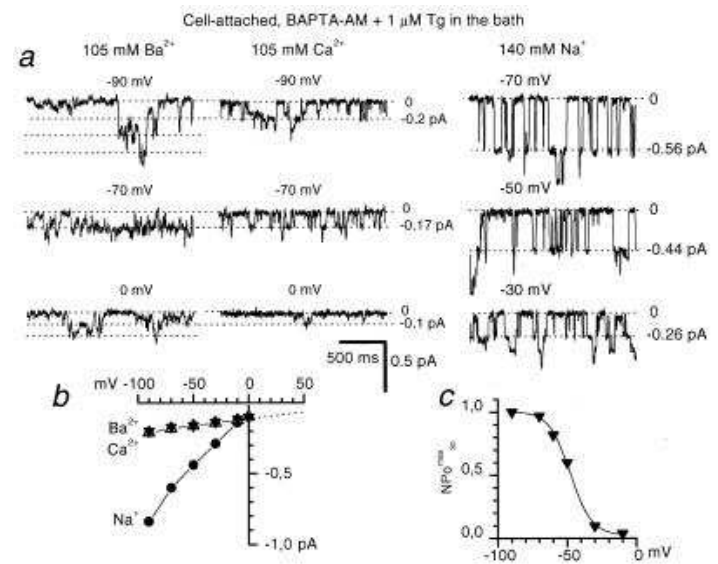

"Conductance properties of store-operated channels in A431 cells. (a) Store-operated channels in A431 cells, activated by the mixture of $100 \mathrm{mM}$ BAPTA-AM and 1 $\mathrm{mM} \mathrm{Tg}$ in the bath solution, were recorded in c/a mode with $105 \mathrm{mM} \mathrm{Ba} 2+$ (Left), $105 \mathrm{mM} \mathrm{Ca} 2+$ (Center), and $140 \mathrm{mM} \mathrm{Na}+$ (Right) in the pipette solution at membrane potential as indicated. (b) Fit to the unitary current-voltage relationship of store-operated channels with $\mathrm{Ba} 2+(\mathrm{n}=46), \mathrm{Ca} 2+(\mathrm{n}=4), \mathrm{Na}+(\mathrm{n}=3)$ yielded slope single-channel conductance of $1 \mathrm{pS}$ for $\mathrm{Ca} 2+$ and $\mathrm{Ba} 2+$ and $6 \mathrm{pS}$ for $\mathrm{Na}+$. (c) Open channel probability of storeoperated channels (NPomax30) expressed as a function of membrane potential. Data from six independent experiments in c/a mode with $105 \mathrm{mM} \mathrm{Ba}+$ as a current carrier were averaged at each membrane potential. (b and c) The average values are shown as mean \pm SEM, unless the size of the error bars is smaller than the size of the symbols."

Figure 10. Above, caption text and image of a MIX figure incorrectly labeled as GRAPH by Image Classifier and GEL_IMAGE by the Text Classifier

\section{Acknowledgements}

We thank three anonymous reviewers for their valuable comments. Hong $\mathrm{Yu}$ and Minsuk Lee acknowledge the support of JDRF 6-2005-835.

\section{References}

Anil K. Jain and A. Vailaya., August 1996, Image retrieval using color and shape. Pattern Recognition, 29:1233-1244

Anil K. Jain, Fundamentals of Digital Image Processing, Prentice Hall, 1989

C. J. van Rijsbergen. Information Retrieval. Butterworths, London, second edition, 1979.

Joachims T, 1998, Text categorization with support vector machines: Learning with many relevant features.
Presented at Proceedings of ECML-98, 10th European Conference on Machine Learning

Kou, Z., W.W. Cohen and R.F. Murphy. 2003. Extracting Information from Text and Images for Location Protemics, pp. 2-9. In ACM SIGKDD Workshop on Data Mining in Bioinformatics (BIOKDD).

Murphy, R.F., M. Velliste, J. Yao, and P.G. 2001. Searching Online Journals for Fluorescence Microscope Images depicting Protein Subcellular Location Patterns, pp. 119-128. In IEEE International Symposium on Bio-Informatics and Biomedical Engineering (BIBE).

Murphy, R.F., Kou, Z., Hua, J., Joffe, M., and Cohen, W. 2004. Extracting and structuring subcellular location information from on-line journal articles: the subcellular location image finder. In Proceedings of the IASTED International Conference on Knowledge Sharing and Collaborative Engineering (KSCE2004), St. Thomas, US Virgin Islands, pp. 109-114.

Sable, C. and V. Hatzivassiloglou. 2000. Text-based approaches for non-tropical image categorization. International Journal on Digital Libraries. 3:261-275.

Sable, C., K. McKeown and K. Church. 2002. NLP found helpful (at least for one text categorization task). In Proceedings of Empirical Methods in Natural Language Processing (EMNLP). Philadelphia, PA

Sable, C. 2003. Robust Statistical Techniques for the Categorization of Images Using Associated Text. In Computer Science. Columbia University, New York.

Sussman J.L., Lin D., Jiang J., Manning N.O., Prilusky J., Ritter O., Abola E.E. (1998) Protein Data Bank (PDB): Database of Three-Dimensional Structural Information of Biological Macromolecules. Acta Crystallogr D Biol Crystallogr 54:1078-1084

MATLAB TM. The Mathworks Inc., http://www.mathworks.com/

Weston, J., A. Elisseeff, G. BakIr, F. Sinz. Jan. 26 ${ }^{\text {th }}$, 2005. The SPIDER: object-orientated machine learning library. Version 6. MATLAB Package. http://www.kyb.tuebingen.mpg.de/bs/people/spider/

Wurst, M., Word Vector Tool, Univeristät Dortmund, http://www-ai.cs.unidortmund.de/SOFTWARE/WVTOOL/index.html 